Rev. Salud pública. 8 (1): 52-62, 2006

ARTÍCULOSIINVESTIGACIÓN

\title{
Escala para medir la Calidad de la Recuperación Postanestésica desde la Perspectiva del Usuario
}

\author{
Javier Eslava-Schmalbach ${ }^{\mathrm{I}}$, Hernando Gaitán-Duarte ${ }^{\mathrm{II}}$ y Carlos Gómez-Restrepo ${ }^{\mathrm{III}}$ \\ ${ }^{\text {I }}$ Médico Cirujano. M. Sc. Epidemiología Clínica. Anestesiólogo. Unidad de Anestesiología e Instituto de \\ Investigaciones Clínicas, Universidad Nacional de Colombia. E-mail: jheslavas@unal.edu.co \\ II Médico Cirujano, M. Sc. Epidemiología Clínica. Gineco-Obstetra. Departamento de Gineco-obstetricia e \\ Instituto de Investigaciones Clínicas, Universidad Nacional de Colombia. E-mail: hggaitand@unal.edu.co \\ III Médico. M. Sc. Epidemiología Clínica. Psiquiatra. Departamento de Psiquiatría y Salud Mental y \\ Unidad de Epidemiología Clínica, Pontificia Universidad Javeriana. E-mail: cgomez@javeriana.edu.co
}

Recibido 9 Febrero 2005/Enviado para Modificación 11 Diciembre 2005/Aceptado 14 Enero 2006

\section{RESUMEN}

Objetivo Desarrollar una escala válida para medir la calidad de recuperación postanestésica desde la perspectiva de los pacientes (CdR).

Métodos Se realizó en sujetos programados para cirugía electiva con clasificación ASA I o II. Lugar: Hospital San Juan de Dios de Bogotá, Clínica de Salud Sexual y Reproductiva Profamilia y Clínica Carlos Lleras del Seguro Social. Se realizó en cinco fases: I. Entrevistas semiestructuradas para conocer las diferentes categorías e ítem de calidad referidos por los pacientes $(n=30)$; II. Categorización y análisis de contenidos para conocer los ítem relevantes $(n=42)$; III. Validación de apariencia $(n=20)$. IV. Diseño y validación de la escala, CdR ( $n=283)$ : Análisis de factores principales, rotación varimax. La consistencia interna se evaluó con el coeficiente Alpha de Cronbach. La validación de criterio, concurrente y discriminante se hizo utilizando estadística no paramétrica; $V$. Evaluación de reproducibilidad de la escala $(n=100)$, mediante el coeficiente de concordancia de Kendal.

Resultados CdR se construyó en español colombiano, con 14 ítems y 3 dominios, calidad general (8 ítems), sala de recuperación (5 ítems) y dolor (1 ítems). Mostró buena consistencia interna (Cronbach $=0.8783)$. $\mathrm{CdR}$ se correlacionó positivamente (rho spearman>0.39) con 3 de 5 escalas. Discriminó diferencias por sexo, tipo de cirugía, sitio quirúrgico y tipo de anestesia (Ji2 y K. Wallis, $p<0.05$ ). Tuvo buena reproducibilidad (Kendal $=0,6378$, $\mathrm{p}=0,033)$.

Conclusión CdR es la primera escala válida para medir la calidad de la recuperación postanestésica, construida sólo desde la perspectiva de los pacientes. 
Ferreira - Malaria incidence

Palabras Clave: Período de recuperación de la Anestesia, calidad de la atención, salud, satisfacción del paciente (fuente: DeCS, BIREME).

\section{ABSTRACT \\ A scale for measuring the quality of post-anaesthetic recovery from the user's point of view}

Objective Developing a valid scale for measuring the quality of post-anaesthetic recovery (QoR) from the patients' point of view.

Methods ASA I or II scheduled surgical patients were selected. Setting: Hospital San Juan de Dios, Bogotá, Profamilia's Sexual and Reproductive Health Clinic and Social Security's Clínica Carlos Lleras. Five phases: semistructured interviews for establishing different quality categories and items referred by patients $(n=30)$; content analysis and categorisation to establish relevant items $(n=42)$; facial validity $(n=20)$; scale design and validation, QoR $(n=283)$ : main factor analysis, varimax rotation Cronbach's alpha coefficient was used for testing internal consistency. Concurrent and discriminating criteria were validated by using non-parametrical statistics]; and reproducibility $(n=100)$, tested by Kendal's concordance coefficient.

Results The QoR was constructed in Colombian Spanish with 14 items and 3 domains, general quality (8 items), recovery room (5 items) and pain (one item). The QoR showed good internal consistency (Cronbach $=0.8783)$. The QoR positively correlated (Spearman's rho>0.39) with 3 out of 5 scales and found differences by gender, kind of surgery, surgical site and kind of anaesthesia ( $\mathrm{Ji} 2$ and $\mathrm{K}$. Wallis, $\mathrm{p}<0.05$ ). The QoR had good reproducibility (Kendal $=0.6378, \mathrm{p}=0.0335$ ).

Conclusion The QoR is the first valid scale constructed from the patient's point of view for measuring the quality of post-anaesthetic recovery.

Key Words: Anesthesia recovery period, quality of health care, patient satisfaction (source: MeSH, NLM).

4 n anestesiología, la mayoría de las mediciones de los indicadores utilizados para evaluar la calidad de la recuperación postanestésica han involucrado, la percepción del personal de salud más que la percepción de los pacientes. Esto ha llevado al uso de indicadores centrados en variables de resultado como el vómito, escalofrío, nauseas, tiempo para despertar, etc. Son escasos los estudios en los que se ha considerado la percepción de los pacientes en lo que se considera una buena calidad de la recuperación postanestésica. Algunos se han hecho desde la perspectiva cualitativa general $(1,2)$, y sólo uno es aplicable al campo de la recuperación postanestésica (Quality of Recovery, QoR), e incluye las percepciones de los pacientes, de los familiares, médicos y enfermeras (3). Los otros se aplican en el campo del cuidado intensivo (4), en el ámbito de la cirugía odontológica 
con anestesia local (5), o en las percepciones postoperatorias de la cirugía de reducción de mama (6). Por tanto, no existe una escala válida que mida bajo la perspectiva única del paciente, la calidad de la recuperación postanestésica.

El objetivo de este trabajo es desarrollar una escala válida que permita medir la calidad de la recuperación postanestésica utilizando únicamente la perspectiva de los pacientes, programados para cirugía electiva, con calificación del Estado Físico de la American Society of Anesthesiology, (ASA) I o II.

\section{MATERIAL Y MÉTODOS}

La realización de este trabajo se acogió a las recomendaciones locales e internacionales para investigación en seres humanos. A todos los individuos se les solicitó consentimiento informado verbal y para las Etapas III a VI verbal y escrito. Esta investigación tuvo aprobación de un Comité de Ética Institucional.

I Fase: Identificación de categorías e ítem propuestos por los pacientes. Pacientes del programa de cirugía ambulatoria en las primeras dos instituciones hospitalarias involucradas, 18 y 12 sujetos respectivamente. Los pacientes fueron elegidos por conveniencia. Se les aplicó entrevista semi-estructurada con el fin de obtener las categorías, de lo que significaba la calidad de la recuperación postanestesica, en tres momentos de atención: en la valoración preanestésica; en el post-operatorio inmediato de cirugía ambulatoria (4 horas); y, en el post-operatorio tardío (8 días)

II Fase: ponderación de la relevancia de los ítems.

Con el listado de los ítems sugeridos en la primera etapa, se buscó la relevancia de los mismos en un nuevo grupo de pacientes, en la recuperación postanestésica Se utilizó una escala verbal análoga, de cero a diez. Adicionalmente se les preguntó a los individuos sobre el valor entre 0 y 10, a partir del cual ya un ítem no era relevante, para definir el punto de corte.

III Fase: validez de apariencia de los ítems.

Con la modalidad de la doble entrevista, en un nuevo grupo de pacientes $(n=20)$, se indagó sobre dos tipos diferentes de fraseo de la misma pregunta. Se eligió la opción que tuviera mayor número de respuestas a favor. 
IV Fase: Diseño de la escala

Análisis de factores principales, rotación varimax, para estructurar dominios y reducir ítems. Se incluyeron 283 pacientes, del postoperatorio inmediato, de la tercera institución involucrada, con la complejidad de un segundo nivel de atención. La evaluación de consistencia interna se hizo mediante el coeficiente alpha de Cronbach.

V Fase: validación de la escala Se realizó con los mismos sujetos de la fase IV. Se comparó CdR con: QoR, la escala visual análoga de la recuperación (VAS-REC), la Escala Verbal Análoga de la satisfacción con la recuperación (VERB), la Sumatoria de Ítems verbales análogos (SUMITEM) y de la Sumatoria total de la escala original (SUMORIG). Para la validación discriminante se compararon las puntuaciones de la escala, por tipo de cirugía (ambulatorios, de corta estancia y de más de dos días de estancia), por sexo, edad, el sitio de la cirugía (cabeza, cuello, tórax, abdomen, miembros superiores, miembros inferiores, periné, otros), el tipo de anestesia (general, regional o combinada), el manejo de la vía aérea (cánula nasal, mascara facial, mascara laringea, intubación endotraqueal), la calificación de Estado Físico (ASA), y el riesgo quirúrgico (John Hopkins) (7). Se utilizó estadística no paramétrica dado que las variables no tuvieron distribución normal. Las variables ordinales se compararon mediante la Rho de Spearman, y la prueba U de Mann-Whitney con la escalas categorizadas (equivalente a $>=8 / 10=1,<8=0$ ). La concordancia entre escalas se evaluó con la prueba kappa. El análisis de la capacidad discriminante de CdR se hizo buscando diferencias en las puntuaciones por sexo (Ji2), Tipo de Cirugía, Sitio Quirúrgico, Tipo de Anestesia y Vía Aérea (Kruskal Wallis), y la edad, Estado Físico, ASA y Riesgo Quirúrgico mediante la U de Mann Whitney. Adicionalmente se hizo una evaluación con los datos extremos.

Se realizaron las siguientes "preguntas de control”, que evaluaron de manera global la calidad de la recuperación de la anestesia: Pregunta 1: Si lo tuvieran que volver a operar le gustaría que le volvieran a hacer todo lo mismo, para tener esta recuperación: Si, No estoy seguro, Definitivamente No; Pregunta 2: Se siente como si no lo hubieran operado: Si, No estoy seguro, Definitivamente No; Pregunta 3: Califique qué tanto se sintió afectado por dolor en la herida cuando despertó, de 0 a 10, siendo 0 nada y 10 mucho. Las puntuaciones de las escalas fueron comparadas con las respuestas a estas preguntas. Se utilizó la prueba de Kruskal Wallis (preguntas 1 y 2) y la prueba rho Spearman (pregunta 3). Adicionalmente, se hizo una compara- 
ción entre el tiempo quirúrgico y el anestésico con cada una de las puntuaciones de las escalas (rho Spearman)

VI: etapa: variabilidad test-retest Se aplicó CdR, sobre el mismo paciente, en dos oportunidades, a las 4 y 6 horas del periodo postoperatorio $(\mathrm{n}=100)$. La escala fue aplicada por dos sujetos diferentes, cada una de ellos ciego a los resultados de la evolución previa. Se utilizó el coeficiente de concordancia de Kendal. En todas las Etapas se utilizó el paquete estadístico STATA versión 6.0.

\section{RESULTADOS}

De un total de 138 ítems iniciales se obtuvieron 43 ítems después de evaluar la mayor relevancia por parte de los encuestados, eliminar preguntas repetidas y frasear la pregunta de la manera que mejor interpretara la opinión de los pacientes.

Se analizaron finalmente 266 de los 283 inicialmente incluidos para la fase de análisis factorial. La edad promedio fue de 37 años con rango entre 15 y 86 años. La razón de feminidad fue de 3.9. El tipo de cirugía fue predominantemente ambulatoria en 199 sujetos (74,8 \%), 39 tuvieron procedimientos de corta estancia (14,6\%), y 28 sujetos estuvieron hospitalizados más de un día $(10,5 \%)$. La mayoría de procedimientos fueron realizados en abdomen (65 \%), en miembros inferiores (12,4\%), y en región perineal $(6,7$ $\%)$. Los pacientes fueron sometidos a anestesia general en su mayoría (86 $\%$ ), a anestesia regional (11,2 \%), y sólo 2,6 \% recibieron anestesia combinada. El 83,2 \% de los pacientes fueron ASA I y el 16,7 \%, ASA II. El 89,2 \% fueron clasificados como Riesgo Quirúrgico I, y el 10,7 \% como Riesgo Quirúrgico II.

Con los resultados del análisis factorial, para el diseño de la escala, se seleccionaron los 5 primeros factores que representaron el 86,2 \% de la varianza. La escala (CdR) presentó una consistencia interna alta (Alpha de Cronbach: 0,8783) (Tabla 1). La escala se construyó con 14 ítems, de carácter ordinal, entre 1 y 5 . La mínima puntuación posible fue de 14 y la máxima de 70 . Entre 1 y 5 se calificó el nivel de satisfacción del paciente con el ítem evaluado.

Validación de criterio

La comparación de las escalas mostró una alta correlación (rho de Spearman) entre CdR con la mayoría de escalas (SUMITEM rho=0.3995 
$\mathrm{p}<0.0005$, VERB rho $=0.3160 \mathrm{p}<0.0005$, SUMORIG rho $=0.8568 \mathrm{p}<0.0005$ ), a excepción de QoR (rho=0.05 p=0.34) y la escala visual análoga (rho=0.20 $\mathrm{p}=0.0011$ ). QoR no se correlacionó con ninguna de las escalas contra las que se comparó (rho $<0.2 \mathrm{p}>0.05)$. El nivel de concordancia entre las escalas en el nivel de satisfacción alto (mayor o igual a 8/10) mostró un buen acuerdo para CdR con SUMITEM (kappa $=0.2763 \mathrm{p}<0.0005)$, con VERB (kappa $=0.2914 \mathrm{p}<0.0005)$, VAS-REC (kappa $=0.1976 \mathrm{p}=0.0006)$ y SUMORIG (kappa=0.7729 $\mathrm{p}<0.0005$ ). QoR tuvo un nivel de acuerdo con SUMITEM superior al esperado por azar, con un kappa bajo (kappa $=0.0514$, $\mathrm{p}=0.0214$ ). No hubo concordancia entre CdR y QoR (kappa=0.042, $\mathrm{p}=0.45$ ). Con respecto a las 3 preguntas de control de la calidad de la recuperación se obtuvo los resultados que se presentan en la Tabla 2. Estas diferencias fueron también clínica y estadísticamente significativas, especialmente para CdR. Hubo una correlación entre $\mathrm{CdR}$ con la duración de la cirugía (rho=0,39 $\mathrm{p}=0,0000)$ y de la anestesia (Spearman rho=0,369 $\mathrm{p}=0,0000)$. Dicha correlación fue baja (Spearman rho $<0,25$ ) para QoR, SUMITEM, VERB, VAS-REC y SUMORIG.

Tabla 1. Valores del Alpha de Cronbach y Correlación Inter-ítem luego de eliminar variables de baja correlación

\begin{tabular}{|c|c|c|c|c|}
\hline Item & $\begin{array}{l}\text { Correlación } \\
\text { Ítem-prueba }\end{array}$ & $\begin{array}{l}\text { Correlación } \\
\text { Ítem-resto }\end{array}$ & $\begin{array}{l}\text { Correlación } \\
\text { Inter-item }\end{array}$ & Alpha \\
\hline $\mathrm{CH}$ del anestesiólogo & 0.7366 & 0.6768 & 0.3274 & 0.8635 \\
\hline CH de enfermería & 0.6990 & 0.6326 & 0.3316 & 0.8658 \\
\hline $\mathrm{CH}$ del cuerpo medico & 0.7636 & 0.7088 & 0.3244 & 0.8619 \\
\hline $\mathrm{CH}$ de todos en general & 0.7386 & 0.6791 & 0.3272 & 0.8634 \\
\hline CA de enfermería & 0.7723 & 0.7190 & 0.3234 & 0.8614 \\
\hline CA en general & 0.7140 & 0.6500 & 0.3300 & 0.8649 \\
\hline CA en recuperación & 0.7210 & 0.6583 & 0.3292 & 0.8645 \\
\hline Atención de los médicos & 0.5922 & 0.5094 & 0.3436 & 0.8719 \\
\hline Ver rápido parientes & 0.5728 & 0.4876 & 0.3457 & 0.8729 \\
\hline Información suficiente & 0.5668 & 0.4810 & 0.3465 & 0.8733 \\
\hline Observación heridas & 0.4845 & 0.3885 & 0.3552 & 0.8774 \\
\hline Valoración física constante & 0.5746 & 0.4896 & 0.3455 & 0.8728 \\
\hline Anestesiólogo Recuperación & 0.4939 & 0.3995 & 0.3546 & 0.8772 \\
\hline Dolor en la herida* & 0.2818 & 0.1713 & 0.3783 & $0.8878^{*}$ \\
\hline Prueba & & & 0.3402 & 0.8783 \\
\hline
\end{tabular}

*No se excluye dolor por percepción de su influencia en la calidad de la recuperación

$\mathrm{CH}$ : Calidad Humana; CA: Calidad de Atención

\section{Validez Discriminante}

Hubo una clara diferencia entre los sexos, en los tipos de cirugía, con respecto a si el paciente era ambulatorio, corta estancia u hospitalizado más de un día, situación que fue evidente con CdR, QoR, SUMITEM y SUMORIG (Tabla 3). Con respecto al sitio de la cirugía, existieron diferencias clínicas y estadísticas en las puntuaciones de las escalas CdR (K.Wallis, p=0.0001), 
SUMITEM (K.Wallis, $\mathrm{p}=0.0069$ ) y SUMORIG (K.Wallis, $\mathrm{p}=0.0011$ ). VERB y VAS-REC no discriminaron diferencias en la puntuación por el sitio de la cirugía. Tampoco lo hizo QoR, ni desde el punto de vista clínico, ni estadístico. Las peores puntuaciones para el sitio de la cirugía la tuvieron para CdR, las cirugías en Miembros superiores ( $\mathrm{Me}=53$, IC(95 \%: 49-59.6)) y para QoR, las cirugías realizadas en la cabeza $(\mathrm{Me}=11$, IC(95 \%: 6.0313.4)). CdR, QoR y SUMORIG discriminaron dependiendo de si el paciente recibió anestesia general, regional o combinada. Las puntuaciones en CdR fueron menores en procedimientos bajo anestesia combinada, mientras que para QoR fueron menores para los pacientes que recibieron anestesia regional (Tabla 3). CdR fue la única escala que mostró diferencias por edad (Mann Whitney= 2,90 p=0,0037).

Tabla 2. Tabla de Medianas e IC95\% ( ) en las puntuaciones de las escalas, según respuesta, para cada una de las preguntas $1,2,3^{*}$

\begin{tabular}{ccccccc}
\hline Pregunta & CdeR & QoR & SUMITEM & VERB & VAS-REC & SUMORIG \\
\hline Pregunta1 & 61 & 13 & 119 & 10 & 8 & 187 \\
$\mathrm{Si}$ & $(60-63)$ & $(13-14)$ & $(117-120)$ & $(9-10)$ & $(8-8)$ & $(185-189)$ \\
$\approx \mathrm{NO}$ & 61 & 12 & 113 & 9 & 8 & 185 \\
& $(59-64)$ & $(9.8-13)$ & $(112-116)$ & $(9-9)$ & $(8-8)$ & $(182-187)$ \\
$\mathrm{NO}$ & 51 & 9 & 116.5 & 8 & 7 & 160 \\
& $(44.7-59.0)$ & $(8-12.2)$ & $(81.1-126)$ & $(6.4-10)$ & $(4.7-8)$ & $(147-185)$ \\
k.wallis & $\mathrm{P}=0.004$ & $\mathrm{P}=0.02$ & $\mathrm{P}=0.001$ & $\mathrm{P}=0.0008$ & $\mathrm{P}=0.003$ & $\mathrm{P}=0.001$ \\
\hline Pregunta2. Si & 62 & 13 & 119 & 10 & 8 & 187 \\
& $(61-64)$ & $(12-14)$ & $(116-120)$ & $(9-10)$ & $(8-9)$ & $(185-189)$ \\
$\approx$ NO & 60 & 11 & 116 & 9 & 8 & 185 \\
& $(59-62)$ & $(9.1-13)$ & $(113-119)$ & $(9-10)$ & $(8-8)$ & $(181-187)$ \\
NO & 52.5 & 14 & 112 & 8 & 8 & 170.5 \\
& $(47-61)$ & $(11.9-15)$ & $(97-123)$ & $(7.4-9)$ & $(6-8)$ & $(159-189)$ \\
k.wallis & $\mathrm{P}=0.0002$ & $\mathrm{P}=0.04$ & $\mathrm{P}=0.02$ & $\mathrm{P}=0.001$ & $\mathrm{P}=0.01$ & $\mathrm{P}=0.001$ \\
\hline Pregunta3. $\geq 8$ & 5 & 5 & 5 & 5 & 5 & 5 \\
<8 & $(5-6)$ & $(5-6.0)$ & $(5-6)$ & $(5-6)$ & $(5-6)$ & $(5-6)$ \\
(verbal) & 7 & 6 & 6 & 7 & 6 & 7 \\
Spearman & $\mathrm{Rho=-0.15}$ & $\mathrm{Rho}=-0.08$ & $\mathrm{Rho}=-0.2$ & $\mathrm{Rho}=-0.09$ & $\mathrm{Rho}=-0.07$ & $\mathrm{Rho}=-0.1$ \\
\hline
\end{tabular}

*Pregunta 1: Si lo tuvieran que volver a operar le gustaría que le volvieran a hacer todo lo mismo, para tener esta recuperación. Si $(\mathrm{SI})$, No estoy seguro ( $\approx \mathrm{NO})$, Definitivamente No (NO).

Pregunta 2: Se siente como si no lo hubieran operado. Si (SI), No estoy seguro ( $\approx N O)$, Definitivamente No.

Pregunta 3:Califique qué tanto se sintió afectado por dolor en la herida cuando despertó, de 0 a 10, siendo 0 nada y 10 mucho. Se uso como punto de corte el valor equivalente mayor o igual a 8.

Validación de valores extremos

Se evidenció un nivel de acuerdo entre CdR y las otras escalas, a excepción de QoR, cuando se dicotomizaron para calificaciones de la calidad equivalentes a 9.5 sobre 10 como valor extremo superior (QoR Kappa=0,03 $\mathrm{p}=0.28$; SUMITEM kappa $=0,20 \mathrm{p}=0,0006$; VERB kappa $=0.13 \mathrm{p}=0.001$; VAS-REC kappa=0.12 p=0,01; SUMORIG kappa=0,36 p=0,0000), y 6 so- 
bre 10 como valor extremo inferior, a excepción de QoR y VAS-REC (QoR kappa $=0,007 \mathrm{p}=0.30$; SUMITEM kappa $=1 \mathrm{p}=0,0000$; VERB kappa $=0.2775$ $\mathrm{p}=0,0000$; VAS-REC kappa $=0.07 \mathrm{p}=0,012 ;$ SUMORIG kappa $=0,66$ $\mathrm{p}=0,0000$ ), QoR mostró un nivel de acuerdo significativo únicamente con VAS-REC (kappa=0,13 p=0,014) en el valor extremo superior.

Tabla 3. Medianas e IC95\% para puntuaciones de la calidad de la recuperación según las diferentes escalas, según variables

\begin{tabular}{|c|c|c|c|c|c|c|}
\hline Variable & CdeR & QoR & SUMITEM & VERB & VAS-REC & SUMORIG \\
\hline Sexo $F$ & $\begin{array}{c}63 \\
(61-63)\end{array}$ & $\begin{array}{c}13 \\
(12-13.8)\end{array}$ & $\begin{array}{c}118.5 \\
(116-120)\end{array}$ & $\begin{array}{c}9 \\
(9-10)\end{array}$ & $\begin{array}{c}8 \\
(8-8)\end{array}$ & $\begin{array}{c}186 \\
(185-188)\end{array}$ \\
\hline $\mathrm{M}$ & $\begin{array}{c}56.5^{\star \star} \\
(53.7-59.6)\end{array}$ & $\begin{array}{c}11^{*} \\
(9-13)\end{array}$ & $\begin{array}{c}115.5^{\star \star} \\
(110-117)\end{array}$ & $\begin{array}{l}9 \text { NS } \\
(9-10)\end{array}$ & $\begin{array}{l}8 \mathrm{NS} \\
(8-8)\end{array}$ & $\begin{array}{c}181^{\star \star} \\
(175-187)\end{array}$ \\
\hline $\begin{array}{c}\text { Tipo de } \\
\text { Cirugía } 0\end{array}$ & $\begin{array}{c}62 \\
(61-63)\end{array}$ & $\begin{array}{c}12 \\
(12-14)\end{array}$ & $\begin{array}{c}117 \\
(115-119.7)\end{array}$ & $\begin{array}{c}9 \\
(9-10)\end{array}$ & $\begin{array}{c}8 \\
(8-8)\end{array}$ & $\begin{array}{c}186 \\
(185-188)\end{array}$ \\
\hline 1 & $\begin{array}{c}60 \\
(59-61)\end{array}$ & $\begin{array}{c}9 \\
(8.8-11.2)\end{array}$ & $\begin{array}{c}117 \\
(113-121)\end{array}$ & $\begin{array}{c}10 \\
(9-10)\end{array}$ & $\begin{array}{c}8 \\
(8-8)\end{array}$ & $\begin{array}{c}189 \\
(182-191)\end{array}$ \\
\hline 2 & $\begin{array}{c}58+\dagger \\
(55.9-60.2)\end{array}$ & $\begin{array}{c}13.5 \dagger \\
(12-15)\end{array}$ & $\begin{array}{c}119 \mathrm{NS} \\
(114.9-120)\end{array}$ & $\begin{array}{l}10 \mathrm{NS} \\
(8-10)\end{array}$ & $\begin{array}{l}8 \mathrm{NS} \\
(8-9)\end{array}$ & $\begin{array}{c}177.5 \dagger \\
(172-184)\end{array}$ \\
\hline $\begin{array}{c}\text { Tipo de } \\
\text { Anestesia } 0\end{array}$ & $\begin{array}{c}62 \\
(60.4-53)\end{array}$ & $\begin{array}{c}13 \\
(12-13.8)\end{array}$ & $\begin{array}{c}118 \\
(116-120)\end{array}$ & $\begin{array}{c}9 \\
(9-10)\end{array}$ & $\begin{array}{c}8 \\
(8-8)\end{array}$ & $\begin{array}{c}186 \\
(185-188)\end{array}$ \\
\hline 1 & $\begin{array}{c}59 \\
(57-62)\end{array}$ & $\begin{array}{c}8.5 \\
(6.12-11)\end{array}$ & $\begin{array}{c}117.5 \\
(110-120)\end{array}$ & $\begin{array}{c}10 \\
(9-10)\end{array}$ & $\begin{array}{c}8 \\
(8-8)\end{array}$ & $\begin{array}{c}184.5 \\
(180-189)\end{array}$ \\
\hline 2 & $\begin{array}{c}56+\dagger \\
(47.8-60.3)\end{array}$ & $\begin{array}{c}14 \dagger \dagger \\
(3.8-18)\end{array}$ & $\begin{array}{c}110 \mathrm{NS} \\
(101-119)\end{array}$ & $\begin{array}{c}9 \text { NS } \\
(5.9-10)\end{array}$ & $\begin{array}{c}8 \mathrm{NS} \\
(4.9-8.6)\end{array}$ & $\begin{array}{c}174 \dagger \\
(146-188)\end{array}$ \\
\hline ASA I & $\begin{array}{c}62 \\
(60-63)\end{array}$ & $\begin{array}{c}13 \\
(12-13)\end{array}$ & $\begin{array}{c}117 \\
(115-120)\end{array}$ & $\begin{array}{c}9 \\
(9-10)\end{array}$ & $\begin{array}{c}8 \\
(8-8)\end{array}$ & $\begin{array}{c}186 \\
(185-188)\end{array}$ \\
\hline II & $\begin{array}{l}59 \mathrm{NS} \\
(57-61)\end{array}$ & $\begin{array}{l}11 \text { NS } \\
(9-13.6)\end{array}$ & $\begin{array}{c}118 \mathrm{NS} \\
(113.8-120)\end{array}$ & $\begin{array}{l}10 \text { NS } \\
(9-10)\end{array}$ & $\begin{array}{l}8 \mathrm{NS} \\
(8-8)\end{array}$ & $\begin{array}{c}183 \mathrm{NS} \\
(179.3-188)\end{array}$ \\
\hline $\begin{array}{l}\text { Vía } \\
\text { Aérea }\end{array}$ & $\begin{array}{c}59 \\
(57-62)\end{array}$ & $\begin{array}{c}8 \\
(5.1-11)\end{array}$ & $\begin{array}{c}119 \\
(112-120)\end{array}$ & $\begin{array}{c}10 \\
(9-10)\end{array}$ & $\begin{array}{c}8 \\
(8-8)\end{array}$ & $\begin{array}{c}185 \\
(180-189)\end{array}$ \\
\hline 1 & $\begin{array}{c}64 \\
(63-64)\end{array}$ & $\begin{array}{c}13 \\
(13-14)\end{array}$ & $\begin{array}{c}119 \\
(115-121)\end{array}$ & $\begin{array}{c}9 \\
(9-10)\end{array}$ & $\begin{array}{c}8 \\
(8-9)\end{array}$ & $\begin{array}{c}187 \\
(186-189)\end{array}$ \\
\hline 2 & $\begin{array}{c}57 \\
(52-60)\end{array}$ & $\begin{array}{c}11 \\
(9.2-14)\end{array}$ & $\begin{array}{c}114.5 \\
(108-116)\end{array}$ & $\begin{array}{c}9 \\
(9-10)\end{array}$ & $\begin{array}{c}8 \\
(8-8)\end{array}$ & $\begin{array}{c}180 \\
(173-186)\end{array}$ \\
\hline 3 & $\begin{array}{c}59+\dagger \\
(56-61)\end{array}$ & $\begin{array}{c}12 \dagger \dagger \\
(10-13.8)\end{array}$ & $\begin{array}{c}119 \dagger \\
(116-120)\end{array}$ & $\begin{array}{l}9 \text { NS } \\
(9-10)\end{array}$ & $\begin{array}{l}8 \mathrm{NS} \\
(8-8)\end{array}$ & $\begin{array}{c}183 \dagger \dagger \\
(176-188\end{array}$ \\
\hline $\begin{array}{c}\text { Riesgo } \\
\text { Quirúrg । }\end{array}$ & $\begin{array}{c}61 \\
(60-63)\end{array}$ & $\begin{array}{c}13 \\
(12-13)\end{array}$ & $\begin{array}{c}118 \\
(115-120)\end{array}$ & $\begin{array}{c}9 \\
(9-10)\end{array}$ & $\begin{array}{c}8 \\
(8-8)\end{array}$ & $\begin{array}{c}186 \\
(184-187)\end{array}$ \\
\hline II & $\begin{array}{c}60 \mathrm{NS} \\
(57.2-63)\end{array}$ & $\begin{array}{c}10 \mathrm{NS} \\
(8.6-13)\end{array}$ & $\begin{array}{c}116 \text { NS } \\
(113-120)\end{array}$ & $\begin{array}{l}10 \text { NS } \\
(9-10)\end{array}$ & $\begin{array}{l}8 \mathrm{NS} \\
(8-8)\end{array}$ & $\begin{array}{c}187 \mathrm{NS} \\
(181-189)\end{array}$ \\
\hline . & (- & & & & & $\begin{array}{l}0=\text { aenera } \\
3=\text { intubació } \\
5 ; \dagger+: p<0.05\end{array}$ \\
\hline
\end{tabular}

Reproducibilidad

Las puntuaciones de CdR mostraron las siguientes resultados para la primera y segunda evaluación. Me1= 58 IC95\%(57-59); Me2=57 IC95\% (56.6-58), 
lo que representó un acuerdo significativo entre las dos pruebas (Kendall $=0.637, \mathrm{p}=0,0335$ )

\section{DISCUSIÓN}

La necesidad de conocer la calidad de la recuperación postanestésica desde la perspectiva de los pacientes, exigió la aproximación cualititativa a ellos. Experiencias similares se describen en la literatura, sin que se haya llegado a un proceso final de validación de las mismas $(2,5)$.

Durante el proceso de validación de los ítems, los pacientes hicieron énfasis en lo relacionado con la calidad de atención y con la calidad humana de las personas que los atienden, así como en la posibilidad de ver a los parientes en el periodo de recuperación postanestésica. Esta consideración es fundamental, dado que en muchas de las Unidades de Cuidado Postanestésico (PACU) no se permite el acceso de los parientes.

Otro de los aspectos que resultó relevante para los pacientes fue el haber sido informado de todo lo que iba a suceder, y cómo se había desarrollado el acto anestésico quirúrgico. Adicionalmente el hecho de haber estado anestesiólogo en recuperación. En muchos lugares, en aras de la contención de costos, se evita asignar un anestesiólogo en recuperación de manera permanente.

Recibieron puntuaciones muy bajas, la presencia de signos físicos como el vómito, la borrachera, la nausea, el frío, el escalofrío, etc., de tal forma que no quedaron incluidos para la fase del análisis factorial. Probablemente, el haber desarrollado la escala con pacientes de instituciones de mediana y baja complejidad llevó a que la presentación de estos síntomas en la recuperación no fuera importante, y por lo mismo, poco considerados durante la calificación global de la recuperación postanestésica.

La escala quedó constituida por tres dominios claramente identificables: el de calidad en general, el de la recuperación en sí, y el del dolor. Dentro del dominio de calidad en general los pacientes fueron capaces de discriminar entre calidad humana de las personas que los atiende y la calidad de atención. A diferencia de otras escalas que pretenden medir aspectos relacionados con la recuperación, la escala CdR se concentró más en los aspectos relacionados con el proceso de atención, lo que es una perspectiva realmente novedosa. 
En lo que respecta a la validez de criterio y concurrente, se encontró que CdR discriminó las diferentes categorías de otras variables que pudieran estar relacionadas con la calidad de la recuperación. Desde este punto de vista, la escala CdR tiene criterio para medir la calidad. Igual situación se apreció con QoR, para algunas de las variables utilizadas en la validación de este instrumento, tal cual como lo describen sus autores (3). La diferencia por sexo de CdR, era esperada, dado que muchas de las mujeres incluidas fueron sometidas a procedimientos muy cortos de esterilización quirúrgica, lo que hace que los procedimientos sean muy diferentes por sexo, y por ende el resultado de la recuperación. CdR fue la única escala que encontró las diferencias en calificación por la edad, probablemente relacionado con el tipo de procedimiento que se realizan más en unas edades que en otras.

Con respecto a la ubicación anatómica del procedimiento quirúrgico, CdR reconoció diferencias en el sitio anatómico donde fue realizada la intervención, situación que no discriminó QoR. Para QoR, los autores refieren que encontraron diferencias por ubicación anatómica de los procedimientos quirúrgicos (3). Sin embargo, los procedimientos se realizaron en un hospital de III nivel, por lo que, probablemente, la capacidad discriminatoria de esta escala no se aprecie en instituciones de II nivel. Es una bondad que se le destaca a CdR con respecto a QoR, que puede ser más sensible a discretas variaciones en procedimientos de baja complejidad.

Para QoR fueron menores las puntuaciones para los pacientes que recibieron anestesia regional probablemente por mayor incapacidad física que genera un bloqueo subaracnoideo o peridural, en caso de anestesia regional espinal. CdR detectó diferencias en la puntuación entre ASA Y y ASA II. QoR en este trabajo no pudo detectar dichas diferencias, tal vez porque fue construida originalmente a partir de pacientes ASAIII y ASA IV (3).

Dado que esta escala se diseñó para el postoperatorio inmediato en pacientes de bajo y mediano nivel de complejidad, muchos de ellos fueron ambulatorios, lo que hace muy difícil evaluar la reproducibilidad en un periodo de tiempo mayor. No se puede determinar si hubo persistencia en la memoria de lo contestado en la primera escala cuando se contestó la segunda. De todas formas, la escala pretende evaluar el postoperatorio inmediato que se considera máximo de 6 horas, y dentro de ese margen de tiempo fue en el que se evaluó la reproducibilidad de la misma. El presente trabajo no exploro la sensibilidad la cambio

Entre QoR y CdR hay claras diferencias en su constructo que hace que las dos no sean comparables: QoR es una escala que mide resultados, y CdR 
es una escala que mide el proceso de atención (a excepción del dolor). Ambas discriminan particularidades relacionadas con el manejo de la recuperación, de manera diferente. Será objeto de un estudio posterior, evaluar CdR en el seguimiento postoperatorio mediato y tardío, así como también estudiar su comportamiento en un III nivel de atención, en donde se espera tenga un mayor poder discriminatorio.

En conclusión, CdR es una escala válida que mide la calidad de la recuperación postanestésica, e involucra el proceso de atención, en unidades de bajo a mediano nivel de complejidad •

Agradecimientos. Financiado por la International Clinical Epidemiology Network (INCLEN) y Universidad Nacional de Colombia.

\section{REFERENCIAS}

1. Kern C, Weber A, Aurilio C, Forster A. Patient evaluation and comparison of the recovery profile between propofol and thiopentona as induction agents in day surgery. Anaesth. Intensive Care. 1998; Apr 26 (2): 156-61

2. Hart J, Neiman V, Chaimoff C, Wolloch Y, Djaldetti M. Patient satisfaction in two departments in a community hospital. Isr. J. Med. Sci.1996; Dec. 32(12): 1338-43

3. Myles P, Hunt J, Nightingale C, Fletcher H, Beh T, Tanil D, Nagy A, Rubinstein A, Ponsford J. Development and psychometric testing of a quality of recovery score after general anesthesia and surgery in adults. Anesth. Analg. 1999; Jan 88(1): 83-90

4. Stovsky B, Rudy E, Dragonette P. Comparison of two types of communication methods used after cardiac surgery with endotracheal tubes. Heart Lung 1988; May. 17(3): 281-9.

5. Shugars D, Benson K, White R, Simpson K, Bader J. Developing a measure of patient perceptions of short'term outcomes of third molar surgery. J. Oral Maxilofac. Surg. 1996; Dec. 54(12): 1402-8.

6. Shakespeare V, Postle K. A qualitative study of patients' views on the effects of breast-reduction surgery: a 2-year follow-up survey. Br. J. Plast. Surg. 1999; Apr. 52(3): 198-204.

7. Pasternak LR. Preoperative evaluation of the ambulatory surgery patient. Ambulatory surgery. Anesthesiology Report 1990; 3: 8. 